\title{
ANAPHORES, STRATÉGIES DISCURSIVES ET GENRES TEXTUELS
}

\author{
Brigitte WIEDERSPIEL \\ CELTED, Université Paul Verlaine-Metz. France
}

\begin{abstract}
En): In this paper, we start with the fact that every text presents a dominating shape of anaphoric relations. We wish to experiment the communicative dynamism of anaphora in two text genres: scientific written one versus essay. Our analysis leads to these conclusions: (1) the author of scientific written text realizes its objective aim in accordance with a centrifugal homotopy and the dynamism put the topic in title to be expanded towards descriptive spaces; (2) in a different way, the author of essay conceptualizes, deforms or manipulates factual data by directing them to a creative focus, in accordance with a centripetal homotopy.
\end{abstract}

Keywords (En): Anaphora; homotopy; text genres; conceptualization; strategy.

\section{Problématique}

Nous partons d'un constat: chaque texte présente une forme prépondérante de rappel anaphorique. Dans ce travail, nous n'entendons pas dresser une énième classification des types et genres textuels mais plutôt, en nous appuyant sur les classifications hétérogènes existantes, explorer par le jeu des anaphores démonstratives observées (désormais AD), les stratégies discursives et les dynamiques informationnelles caractéristiques dans deux genres différents. Nos investigations s'inscrivent en sémantique textuelle et se limitent aux systèmes de la coréférence. L'hypothèse que nous souhaitons défendre s'appuie sur trois postulats de départ :

- $\quad$ Tout d'abord, nous constatons que les systèmes anaphoriques, qui participent à la cohérence interne du texte, varient sensiblement selon les pratiques discursives ${ }^{1}$, nous nous proposons donc de confronter deux genres d'écrits réalisés par des scripteurs experts ou professionnels, l'essai polémique et l'écrit scientifique ${ }^{2}$.

- Ensuite nous considérons comme établi le lien entre relation anaphorique et mémoire discursive : toute anaphore a la faculté de rappeler des données posées ou présupposées du texte, fonction de remémoration, et certaines d'entre elles se valorisent d'un second rôle, fonction de recadrage cognitivodiscursif. Pour cette dernière fonction, l'anaphore indique d'une part une continuité référentielle et, d'autre part, conceptualise le contenu de la reprise, c'est-à-dire le réinterprète, le manipule ou le déforme. Il s'ensuit que l'anaphore participe par cette double propriété à l'identification de fragments de l'information à mémoriser, donc jugés centraux par le sujet épistémique.

- $\quad$ Enfin, cette dernière remarque nous a tout naturellement conduite à examiner de plus près l'impulsion communicative du texte. Dans cette contribution, nous allons pour l'essentiel éprouver l'interdépendance ou l'influence de la

\footnotetext{
${ }^{1}$ Nous suivons ici l'idée défendue par J.-M. ADAM (2010 : 10) selon laquelle « les typologies de rang textuel ne sont pas pertinentes [...] il ne peut s'agir que de types de pratiques discursives, c'est-à-dire de genres ».

2 Quelques linguistes se sont intéressés à d'autres genres dans une optique similaire: Anne CONDAMINES (2005) travaille sur les marqueurs d'hyperonymie dans les manuels spécialisés et Marie VEINARD (2009) sur l'anaphore lexicale dans le discours de Presse.
} 
structure informationnelle du texte sur la structure formelle - niveaux grammatical et sémantico-référentiel - de la proposition.

Nous voulons ainsi défendre l'hypothèse que le système des anaphores démonstratives n'aboutit pas aux mêmes résultats dans les genres textuels exploités - écrits scientifiques et essais polémiques - et que ces différences attendues vont trouver un début d'explication par la prise en compte de la perspective fonctionnelle de le phrase. Nous souhaitons ainsi expérimenter la dynamique sémantico-communicative de l'anaphore.

\section{Méthodologie double}

Pour établir un corpus suffisamment pertinent et représentatif d'AD, nous avons utilisé deux méthodes radicalement différentes selon le genre textuel observé.

- La $1^{\text {ère }}$ méthode est empirique: nous avons en premier lieu fondé nos investigations sur l'examen d'un ouvrage tiré de notre corpus personnel d'essayistes dont le genre argumentatif se prête à la controverse et à l'instauration de cadres discursifs marqués. Cet ouvrage, Du bon usage des catastrophes de Régis DEBRAY (2011; 108 pages, 14953 mots), a été intégralement lu puis scanné, pour permettre la sélection automatique des syntagmes nominaux démonstratifs via un logiciel de traitement de texte. Après avoir éliminé les cataphores, les exophores et autres locutions qui nous paraissent hors propos, comme «à cet égard» par exemple, nous avons comptabilisé 58 AD.

- La $2^{\text {nde }}$ méthode est systémique : nous avons exploité un corpus en ligne, l'outil Scientext, composé de 176 textes scientifiques relevant de quatre disciplines des Sciences Humaines (linguistique, psychologie, sciences de l'éducation et traitement automatique des langues). Cet outil offre des fonctionnalités avancées avec différents modes de recherche : Nous pouvons commenter cette capture d'écran qui le détaille : 


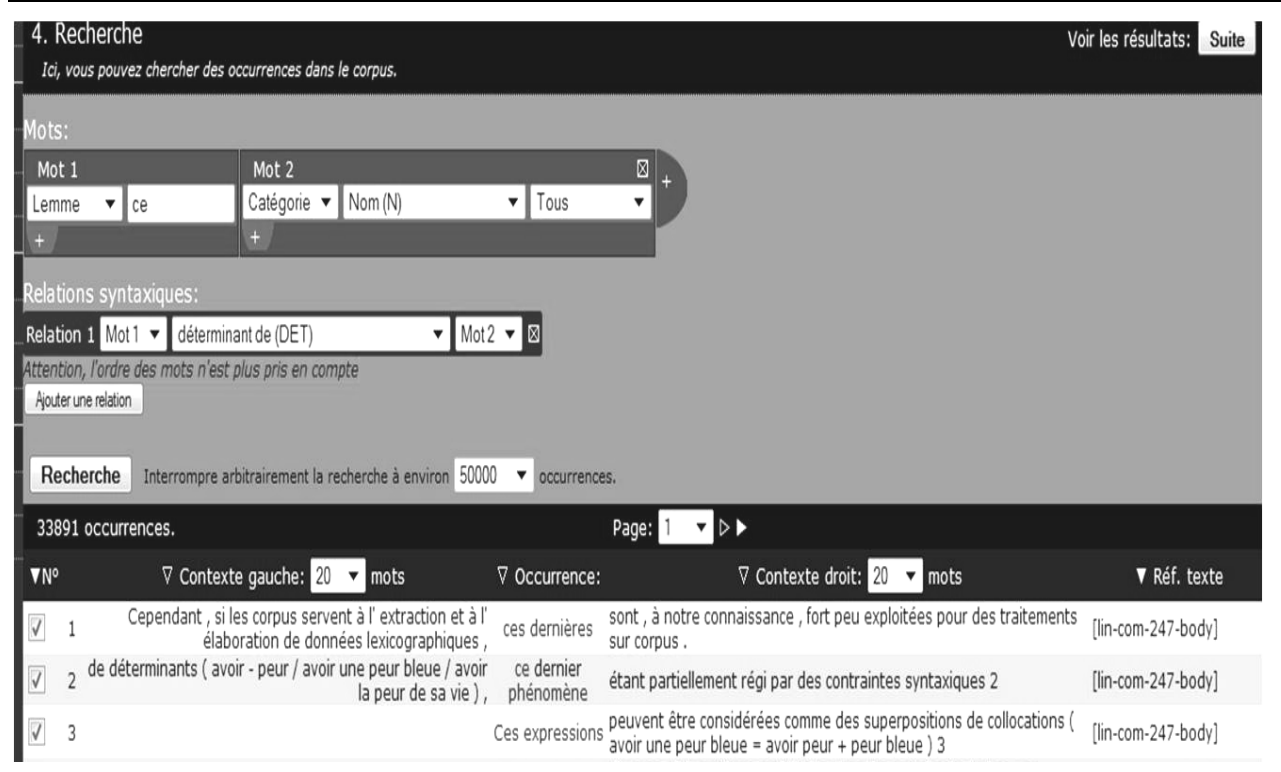

Nous avons utilisé la «recherche libre » qui permet de sélectionner des segments textuels en fonction de leur lemme, ou catégorie et de leurs relations syntaxiques, représentée ici par les deux encadrés supérieurs. La syntaxe que nous avons programmée peut se gloser comme suit : « Extraire du corpus sélectionné toute suite de mots composée d'un nom précédé du lemme «ce » répondant à la relation syntaxique «être le déterminant de »

Nous sommes parvenues au résultat suivant: sur 176 textes scientifiques, Scientext nous a permis d'extraire 33891 syntagmes nominaux démonstratifs, le chiffre variant légèrement à chaque consultation selon les mises à jour du site. Le relevé des occurrences de SN démonstratifs est indiqué sous la colonne centrale «Occurrence». Chaque résultat se trouve doublement indexé dans la dernière colonne de droite: sous-catégorisant d'une part les types d'écrits scientifiques (article, communication, thèse ou habilitation) et localisant d'autre part l'occurrence relevée (introduction, corps du texte, conclusion, note, résumé, etc.). Ces références sont reproduites à la fin de chaque exemple cité. Comme précédemment, il a fallu isoler à bon escient les véritables $\mathrm{AD}$ des syntagmes démonstratifs non anaphoriques.

\section{Mises au point préalable}

\subsection{Premier préalable : définition de l'anaphore résomptive conceptuelle ou ARC}

Nous devons tout d'abord rappeler les fondements de l'ARC, catégorie nouvelle d'anaphore qui demande à être stabilisée et qui entend répondre à ce type de questionnement : comment reprendre du déjà dit tout en introduisant un élément nouveau qui relève de la manière de voir du scripteur?

Prenons immédiatement un extrait de Régis Debray suffisamment représentatif de ce phénomène: 
(1) La désertion des lieux de culte ouvre la porte aux novices qui-ne-remplissent-pas-lescritères pour qu'ils prennent l'angoisse en charge, en lieu et place des anciens gestionnaires. Ainsi les pouvoirs publics sont-ils contraints d'expédier des brigades de psys (les cellules médico-psychologiques) sur les lieux de l'avalanche ou de l'attentat, parce que le curé comme l'instituteur ne sont plus opérationnels pour ce qui est de donner un sens au deuil et à la mort. Ces suppléances sont à la charge du contribuable et il faudra bien qu'un économiste néolibéral chiffre en matière de sécurité civile les coûts budgétaires de la déchristianisation, comme de la fin des religions civiles de salut. Quand la Vierge ou saint Christophe ne protègent plus contre les accidents de la circulation, quand les réducteurs d'incertitude que furent les rogations, le cierge et l'ex-voto, puis Le Capital et le Comité central deviennent folkloriques, chacun s'en va signer un contrat auprès des compagnies d'assurances et réclamer des indemnités auprès des cabinets d'avocat. (DEBRAY : 63)

Nous qualifions «ces suppléances » comme étant une ARC dans la mesure où elle résume et condense la séquence et amène de surcroît un point de vue singulier sur l'origine des cellules médico-psychologiques. Nous glosons le passage ainsi :

«Les brigades de psy sont envoyées par les pouvoirs publics lors d'événements potentiellement traumatiques. Mais ce ne sont que des novices qui-ne-remplissent-pas-lescritères pour donner un sens au deuil et à la mort. Ces institutions, tout comme les compagnies d'assurance et les avocats, se sont imposées depuis la désertion des lieux de culte et la fin des religions civiles de salut. Ce sont des suppléances»

Le nom-tête «suppléances » procède d'un travail de recatégorisation, de remaniement conceptuel sur la base de traits co-occurrents (en lieu et place, anciens gestionnaires, s'en aller signer) dans les cotextes gauche et droit:il réinterprète négativement les circonstances de l'apparition des cellules de crise. Nous en déduisons que l'interprétation de l'ARC ne se limite pas à la seule reconnaissance d'un élément textuel antécédent, mais résulte d'une interaction entre des objets de mémoire immédiate et des recouvrements présuppositionnels, le tout conduisant à une représentation mentale du centre d'attention local du sujet épistémique, ce que rappelle notre première définition ici reproduite :

«Une séquence première, le plus souvent pluri-prédicative, de nature parfois descriptive, constituée de propositions inductrices à partir desquelles sera réalisée, dans une seconde séquence, la saisie conceptuelle sous la forme, pour le cas général, d'un syntagme nominal démonstratif et pour des cas plus particuliers, d'un syntagme nominal défini qui va mettre à disposition un cadre définitionnel et / ou interprétatif. » (KARA et WIEDERSPIEL, $2010: 204$ )

\subsection{Second préalable : l'Homotopie du Topique et du Focus}

Pour configurer la dynamique sémantico-communicative de l'anaphore, nous avons choisi la perspective fonctionnelle défendue par A. WLODARCZYK (2003) en raison des forces centrifuge et centripète qu'il exploite. Reconnaissant l'apport du Centre Linguistique de Prague, cet auteur propose une théorie générale de la composition des énoncés par le recours à une méthode «représentationnelle » en termes d'espaces ouverts (topologie), avec l'intention de dépasser l'ancienne opposition thème/rhème jugée insuffisante à expliquer le statut linguistique de la structure informative du texte.

Les fondamentaux de sa perspectives sont :

- le concept d'information est un contenu communiqué et validé par le sujet épistémique. 
le Topique et le Focus sont les fragments saillants de l'information, les centres d'intérêt sur lesquels le sujet épistémique souhaite attirer l'attention du lecteur/auditeur. En termes topologiques, ces centres d'intérêt sont des espaces mis en opposition ou encore introduisant un contraste avec des espaces inverses, les commentaires, selon une relation dynamique et continue qui déforme l'information pour la glisser ou la projeter d'un espace à un autre. Cette projection s'appelle l'homotopie :

«L'homotopie est la notion topologique de la déformation continue des espaces ouverts [...] La notion d'homotopie permet de représenter le fait que le centre d'intérêt n'est pas un point ou espace fixe mais bien une opération construisant de façon dynamique deux types d'espaces inverses : l'un qui est le centre d'intérêt centrifuge (topique) partant d'un point et se projetant sur un espace, l'autre qui est le centre d'intérêt centripète (focus) partant d'un espace et se projetant sur un point. » (WLODARCZYK, $2003: 522-523$ )

Le rapport inverse des deux parties d'un message s'analyse comme suit; si le centre d'intérêt est connu et le commentaire nouveau, il s'agit d'un énoncé à topique ; si le centre d'intérêt est nouveau et le commentaire connu, il s'agit d'un énoncé à Focus, comme le schématise cette figure sur l'expansion et la contraction des espaces du Topique et du Focus présentée par WLODARCZYK (2003: 523) :

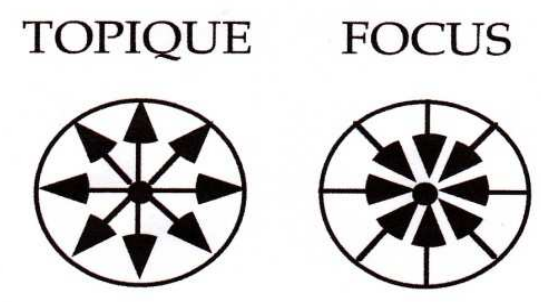

Nous pouvons à présent esquisser un tableau synoptique des réalisations anaphoriques ${ }^{3}$ observées dans les deux genres textuels et du rapport inverse que tend à montrer leur dynamique communicative.

\footnotetext{
${ }^{3}$ Dans cette perspective, nous nous limitons au système de la coréférence et laissons de côté les anaphores divergentes qui présentent soit des relations associatives ou présuppositionnelles (Cf. les travaux de Mathilde Salles) soit d'Elaboration (Cf. les discussions de Georges Kleiber et Hélène Vassiliadou).
} 
Anaphores, stratégies discursives et genres textuels

\begin{tabular}{|c|c|c|c|c|}
\hline \multirow{2}{*}{$\begin{array}{l}\text { Genres } \\
\text { textuels }\end{array}$} & \multirow[b]{2}{*}{ Homotopie } & \multicolumn{3}{|c|}{ Types d'anaphores démonstratives } \\
\hline & & AD type fidèle & AD type ARC & $\begin{array}{c}\text { Autres AD } \\
\text { coréfentielles }\end{array}$ \\
\hline 窇 & $\begin{array}{c}\text { Expansion } \\
\text { Sélection d'un centre } \\
\text { d'intérêt connu ; } \\
\text { Multiplication de la } \\
\text { mention des référents } \\
\text { par des séries de } \\
\text { reprise à l'identique } \\
\text { Enoncés à Topique } \\
\end{array}$ & $\begin{array}{c}\text { Prédominance } \\
\text { des reprises à } \\
\text { l'identique } \\
\text { Anaphore fidèle } \\
\text { Anaphore } \\
\text { résomptive fidèle }\end{array}$ & $\begin{array}{c}\text { Quasi-absence } \\
\text { d'ARC }\end{array}$ & $\begin{array}{c}\text { Nombreuses } \\
\text { Anaphores } \\
\text { résomptives } \\
\text { synonymiques } \\
\text { Rares anaphores } \\
\text { métalinguistiques }\end{array}$ \\
\hline 兽 & $\begin{array}{c}\text { Contraction } \\
\text { Resserrement des } \\
\text { connaissances } \\
\text { factuelles et } \\
\text { inférentielles en une } \\
\text { seule impression } \\
\text { conceptuelle ; } \\
\text { Elaboration d'un } \\
\text { centre d'intérêt. } \\
\text { Enoncés à Focus }\end{array}$ & $\begin{array}{l}\text { Quasi-absence } \\
\text { d'anaphore fidèle }\end{array}$ & $\begin{array}{c}\text { Prédominance des } \\
\text { reprises créatives } \\
\text { ou conceptuelles } \\
\text { ARC } \\
\text { Rares anaphores } \\
\text { métalinguistiques } \\
\text { requalifiantes }\end{array}$ & $\begin{array}{l}\text { Rares anaphores } \\
\text { résomptives } \\
\text { synonymiques } \\
\text { Une occurrence de } \\
\text { SNDP }^{4}\end{array}$ \\
\hline
\end{tabular}

Ainsi, si on accepte la transposition des concepts d'homotopie du Topique et du Focus à notre objet d'étude, il apparaît que ces genres s'opposent radicalement en ces termes :

- le genre descriptif/explicatif /objectivant propre au protocole de rédaction des écrits scientifiques répond à une dynamique communicative de type centrifuge (énoncés à Topique, de l'un vers le multiple ou expansion) réalisée par des séries d'AD marquant fidèlement la continuité discursive des éléments saillants du texte.

- $\quad$ tandis que le genre argumentatif de l'essai polémique présente des types séquentiels différents avec un mouvement communicatif de type centripète (énoncés à Focus, ou contraction sur un point) par la réorientation d'empans textuels subsumés sous l'impression conceptuelle des ARC.

Il nous reste à montrer la conformité de ce tableau synoptique avec les réalisations retenues dans les deux corpus exploités.

\section{Dominante dans les écrits scientifiques : reprises à l'identique}

L'observation de notre $1^{\mathrm{er}}$ corpus a rapidement conduit à l'élaboration des critères distinctifs suivants :

- premièrement, la majeure partie des SN démonstratifs comprennent des noms communs (de type prédicatifs et syncatégorématiques le plus souvent relation, approche, classification, typologie, etc. ${ }^{5}$ )

\footnotetext{
${ }^{4}$ Cf. Les Syntagmes nominaux démonstratifs prédicatifs de Catherine SCHNEDECKER (2006).

${ }^{5} C f$. Le lexique scientifique transdisciplinaire d'Agnès TuTiN (2007).
} 
deuxièmement, un type de réalisation anaphorique se distingue nettement: soit comme anaphore fidèle à l'objet du discours, ou centre d'intérêt explicité en titre ou sous-titre de partie ; soit par une succession d'anaphores fidèles qui développent les différents points ou critères d'une problématique selon un raisonnement scientifique.

Cet exemple est assez représentatif des structures anaphoriques rencontrées. Il nous semble pertinent d'en inférer que la dynamique du niveau informationnel est intimement liée au genre d'écrit :

(2) TITRE : Génération automatique de texte en langue naturelle

$\mathrm{Si}$ la génération automatique est un domaine relativement récent dans le traitement automatique des langues, la nécessité de faire produire des messages par l'ordinateur remonte, elle, aux débuts de l'informatique. Permettre aux différents programmes d'expliciter leurs erreurs, leurs fonctionnements.... à l'utilisateur répond à un véritable besoin. Des messages relativement courts et toujours les mêmes (aFatal ErrorF, ,Core DumpedC...) étaient, et restent encore dans certains cas, considérés comme largement suffisants. Cependant, ces messages ne sont guère explicites, mais surtout s'avèrent mal adaptés aux différentes classes d'utilisateurs. Une première évolution consistait à remplacer ces textes préenregistrés par des phrases également préenregistrées mais comportant des "trous". Ces trous correspondent à des variables qui, lors de la production du message, sont affectées d'une valeur adéquate. (Scientext, [tal-the-21-body] Claude Ponton, Génération automatique de textes en langue naturelle. Essai de définition d'un système noyau)

Nous figurons cette dynamique par un schéma de type centrifuge:

- $\quad$ avec en gras le Topique, les autres segments faisant partie du commentaire.

- le centre d'intérêt connu, ou Topique, «La génération automatique de texte » est introduit dans le titre ${ }^{6}$, c'est-à-dire en position saillante.

- l'expansion rend compte de l'éloignement du centre vers des espaces ou sous-espaces descriptifs et ces espaces ordonnent temporellement le raisonnement. «avant ces messages étaient préenregistrés [...] ensuite, ces textes ont évolués en phrase comportant des trous ».

- $\quad$ chaque espace ou sous-espace marque sa dépendance par une nouvelle série d'anaphore fidèle. « Ces trous correspondent à des variables ».

\footnotetext{
${ }^{6}$ Nous pouvons ici citer la thèse de Marie-Paule JACQUES (2003). Approche en discours de la réduction des termes complexes dans les textes spécialisés, qui spécifie les espaces particuliers du texte dans lesquels l'information doit être particulièrement explicite : l'introduction d'un référent dans ces espaces particuliers permet ensuite au scripteur de l'évoquer sous forme réduite.
} 


\section{Dynamique Centrifuge}

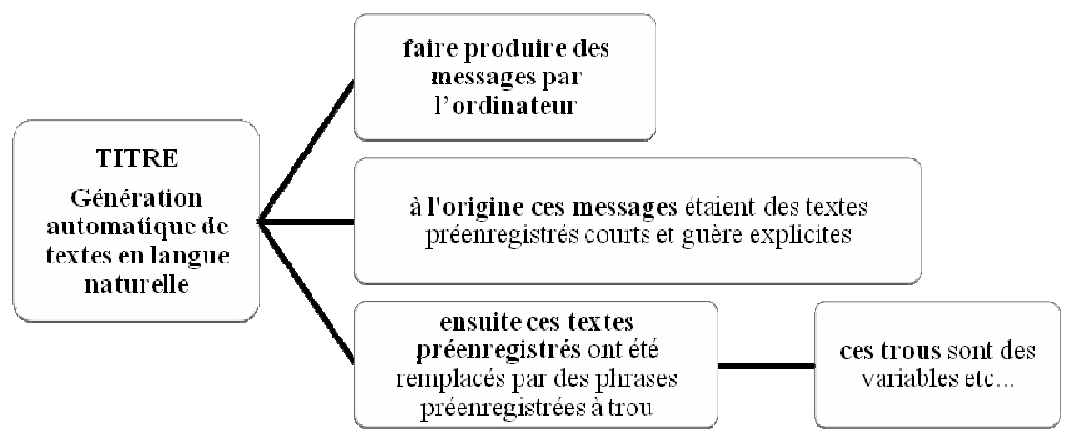

Les configurations en AR sont tout aussi présentes dans les textes scientifiques et réalisent, dans notre perspective, une dynamique sémantico-communicative identique à celle observée avec les anaphores fidèles précédentes. Nous rappelons notre $1^{\text {ère }}$ définition :

« L'AR consiste en la répétition, la reprise et/ou le retraitement d'un thème associé à une ou plusieurs prédications, c'est-à-dire d'une source linguistique non classifiée dans le discours antérieur puisqu'elle n'a pas encore fait l'objet d'une catégorisation nominale, par un SN dont le $\mathrm{N}$-tête est un nom prédicatif qui renvoie à une situation. » (KARA et WIEDERSPIEL, $2010: 203$ )

Nous pensons qu'il est utile ici de distinguer au sein de la catégorie des modes de donation référentielle différents (au sens de Gottlob Frege et réinvestis par Georges Kleiber). Et nous retenons une répartition tripartite des AR. Les 2 premières relèvent des écrits scientifiques, tandis que la dernière est la particularité des essais polémiques.

\begin{tabular}{|lll|}
\hline 3 types d'Anaphore Résomptive & \\
\hline$-\quad$ AR fidèle avec parenté morphologique & Ecrit Scientifique \\
- & AR synonymique & \\
\hline- ARC axiologique & $\longrightarrow$ & Essai polémique \\
\hline
\end{tabular}

Entre AR fidèle et AR synonymique la différence est ténue. Une explication de type structuraliste de l'anaphore conduirait à reconnaître l'existence en langue de trous lexicaux qui bloqueraient la reprise fidèle. Mais nous accordons un autre type d'explication à ces phénomènes. Ainsi dans l'exemple suivant, relevant du champ de la psychologie sous le titre «conflits sociocognitifs et buts d'accomplissement», l'auteur utilise pour condenser l'information « La crainte d'invalidité pousserait à éviter les conclusions prématurées», le nom «motivation ». Ce choix ne se limite pas à la base de traits sémiques co-occurrents mais rend compte de la dynamique interprétative du sujet épistémique. La première $\mathrm{AR}$ « cette motivation » ouvre la voie à un raisonnement en trois étapes marquées par la succession d'AR identiques.

(3) La crainte d'invalidité correspondrait pour sa part à une peur de commettre une erreur coûteuse (KRUGlanski et Freund, 1983). En d'autres termes, elle pousserait à éviter les conclusions prématurées, du fait que celles-ci risquent d'être incorrectes. Cette motivation 
viendrait du coût perçu de l'erreur de jugement. Elle serait en effet à l'oeuvre chez les individus qui estiment qu'ils ne peuvent pas se permettre de se tromper, chez ceux pour qui prime le désir d'exactitude [...]. Là encore, cette motivation peut relever de traits stables comme de la situation (par exemple l'appréhension de l'évaluation, Kruglanski et Freund, 1983). La crainte d'invalidité provoque des réactions inverses à celles que provoque le besoin de clôture. [...]. Contrairement au besoin de clôture, en effet cette motivation favorise la génération d'hypothèses alternatives et la prise en compte des informations contradictoires pour établir son jugement, en vue d'éviter l'engagement trop hâtif dans un jugement qui risque de ne pas être le bon. (Scientext, [psy-the-15-body] Céline Darnon, Conflit sociocognitif et buts d'accomplissement: effets interactifs sur l'apprentissage et le mode de régulation du conflit)

\section{Dynamique Centrifuge}
La crainte d'invalidité correspondrait à une peur de commettre une erreur coutense ...elle pousserait à éviter les conclusions prématurées, du fait que celles-ci risquent d'être incorrectes.

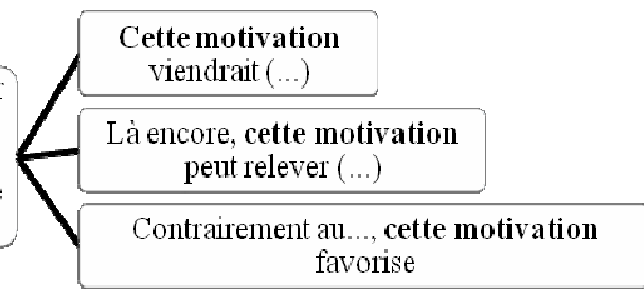

Nous pouvons à présent spécifier le genre «écrit scientifique » dans le champ des Sciences Humaines en particularisant ses deux niveaux sémantico-référentiel et communicatif.

La visée objectivante de tout écrit scientifique se réalise dans des situations discursives particulières qui relèvent de stratégies liées à l'entendement et au partage de la connaissance.

Nous y retrouvons :

- $\quad$ des anaphores fidèles, des AR fidèles et synonymiques qui constituent des successions de reprises à l'identique.

- ces reprises correspondent à une projection, de l'un vers le multiple, c'est-à-dire de l'objet du discours central (ou centre d'intérêt, ou Topique, ou thème local de l'écrit scientifique) généralement placé en position saillante (titre de l'article ou d'une partie) sur des espaces variés et liés par un raisonnement de type scientifique répondant à une progression textuelle propre au genre.

\section{Dominante dans les essais : ARC ou centration idéologique (non dit)}

Pour contribuer à reconnaître l'interdépendance des niveaux formel et informatif du texte, il suffit de montrer qu'un changement de genre textuel conduit forcément à des conclusions sinon inverses du moins différentes que celles observées dans le genre « écrit scientifique».

Nous postulons que le genre «essai » présente des types séquentiels selon une dynamique communicative de type centripète générée par la réorientation d'empans textuels subsumés sous l'impression conceptuelle des ARC. Pour représenter ce genre parmi notre corpus d'essayistes, nous avons choisi de nous concentrer sur l'ouvrage, Du bon usage des catastrophes de Régis Debray (mai 2011). 
Dans l'incipit que nous présentons, l'auteur explique que les catastrophes donnent lieu en retour à trois types de valeur: une valeur d'avertissement, une valeur pédagogique et une valeur de mise en garde contre le catastrophisme. Ces trois retours d'expérience sont subsumés et axiologisés par l'ARC «ces réactions raisonnées au choc émotif, fort salutaire en elles-mêmes ».

(4) Les catastrophes naturelles et industrielles, qui, dans l'agenda de nos effrois, relaient les désastres de la guerre et les pestes d'antan, donnent lieu à maints retours d'expérience, qu'on peut ranger sous plusieurs rubriques également profitables :

$1^{\circ}$ En nous dévoilant l'envers du décor, et la face noire du progrès technique, la catastrophe a valeur d'avertissement. [...] et nous rappelle aux lois de la nature, $[\ldots]$

$2^{\circ}$ En mettant à nu nos imprudences, nos visions à court terme et un fatal esprit de lucre, la catastrophe a valeur de pédagogie: elle nous enjoint de tirer les leçons [...].

$3^{\circ}$ Certains ajoutent, par un autre tour d'écrou, l'alerte à l'alerte, ou la mise en garde contre le catastrophisme, conçu par les experts «écolocrates » du développement durable [...]

Telles sont, outrageusement campées, les diverses façons qu'ont nos nations ménagères de leurs deniers et de leur sang de travailler l'horreur pour tirer le meilleur possible du pire. Avec parfois un tel empressement dans la parade qu'on se sent bientôt dispensé de compassion pour les victimes. Et d'admiration pour les sauveteurs, comme ces «liquidateurs » soviétiques de Tchernobyl envoyés sur les lieux en mission sacrificielle et dont vingt mille sont déjà morts.

Ces réactions raisonnées au choc émotif, fort salutaires en elles-mêmes, ne sauraient cacher un autre type de réponse en pleine recrudescence : l'usage symbolique du désastre par maints responsables politiques, exégètes et commentateurs. Cette sous-traitance par le haut, sans contredire les mesures de protection civile répondant à une demande fort légitime et immédiate de sécurité, répond, elle, à une demande non moins légitime de sens. À une baisse de la production économique, répond, le jour d'après, une hausse de la production prophétique.

(DEBRAY : 11)

On doit noter que l'ARC « Ces réactions raisonnées [...]», sous couvert d'une identité référentielle, permet du même coup d'introduire l'objet du discours les catastrophes dans une conception nouvelle et distincte du «raisonné » et du « salutaire », celle de «l'usage symbolique du désastre » décrite par Régis Debray comme étant la réponse prophétique insufflée par les responsables politiques et les mass média.

Soit encore cet autre exemple plus en avant dans l'ouvrage, où l'ARC « ces brusques transformations d'un substrat physique qu'on appelle une catastrophe naturelle (et qui n'en est une que pour nous) » pose à nouveau une relecture symbolique des catastrophes. L'interprétation du passage est comme toujours chez Régis Debray assez complexe. En effet, le PN anaphorique «en » porte à confusion sur sa saturation référentielle : est-ce une interrogation métalinguistique sur le sens de l'expression, le nom «catastrophe» ou la qualification de « naturelle », ou bien est-ce une interrogation sur l'usage du mot « catastrophe » par le sémiopathe y voit comme un sens caché, un complot une faillite morale?

(5) Sa physiologie fait du bipède sans plumes un mammifère symbolisant, neurologiquement équipé pour donner du sens à tout, y compris à la tuile qui tombe du toit. C'est un malade des signes. Un sémiopathe, à tout instant exposé à des accès de prévisionnite ou de sondomanie, affections chroniques qui ne sont pas des maladies mais des exagérations ou des spécialisations. Ayant l'herméneutique innée et compulsive, il ne peut s'empêcher de voir double. Le sens spirituel ou plénier sous le sens direct et obvie d'un texte consacré. Le contenu caché sous l'enveloppe manifeste des choses. Une faillite morale derrière la catastrophe matérielle. L'idiotie du réel, et notamment de ces brusques transformations d'un substrat 
physique qu'on appelle une catastrophe naturelle (et qui n'en est une que pour nous), lui est psychologiquement insupportable. Plus encore, la brutalité bête des accidents. D'où le caractère prévendu des théories du complot (on nous cache quelque chose) comme des signes des temps (ce n'est pas un hasard si). Ne tolérant pas le mutisme des choses, le premier mouvement de qui n'est pas né de la dernière pluie est de les faire parler. Et pour nous rassurer nous-mêmes sur la valeur de ces aveux, on est malin, on fait le modeste. (DEBRAY : 46)

Tout au long du texte, l'auteur n'aura de cesse d'alimenter une hypothèse qui, contrairement à l'écrit scientifique, n'est pas un objet donné en titre mais constitue le centre d'attention nouveau et créatif à savoir le focus. Nous posons que l'ensemble des ARC de l'ouvrage opèrent une réorientation d'espaces factuels dans un nouvel espace, celui relatif à la théorie du retour au prophétisme. On se risquera à synthétiser cette créativité comme suit :

«L'auteur entend défendre l'idée qu'aujourd'hui, les chefs d'état, les gouvernements, les commissions européennes, et les communicants utilisent toujours la réponse prophétique pour donner du sens à toute catastrophe (naturelle, nucléaire, informatique, technologique, etc.). »

La figure suivante schématise la contraction opérée à partir de plusieurs connaissances factuelles, inférentielles connues (les catastrophes, les doctrines et le pratiques sociales) en une seule impression conceptuelle nouvelle (le prophétisme). La dynamique informationnelle réalisée est une focalisation sur un centre d'intérêt centripète qui part de sous-espaces pour se projeter vers un point central.

\section{Dynamique centripète}

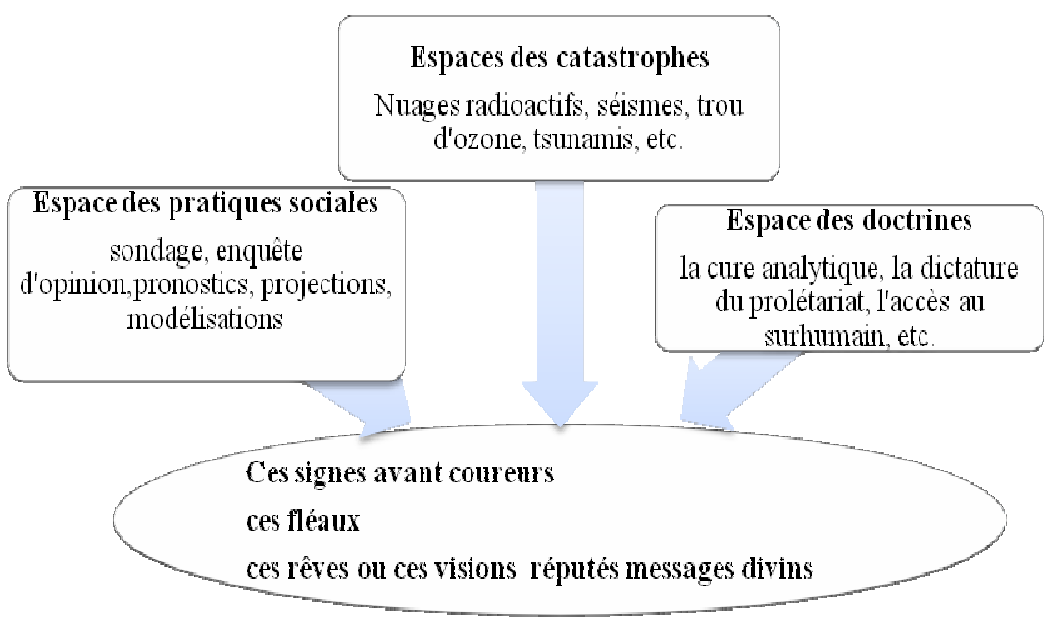

Ainsi les différentes ARC de l'ouvrage déforment les catastrophes en "fléaux ou signes avant-coureurs », réorientent les rêves en « visions » et réinterprètent les sondages ou autres pronostics comme des augures de la vox populi.

Enfin, notre proposition est corroborée par le fait même que les AR métalinguistiques de ce texte sont requalifiantes et deviennent par là-même déformatives, contrairement à celles des écrits scientifiques. Elles assument ainsi 
une triple fonction: découpage du matériau linguistique, catégorisation d'une forme de discours et requalification en contenu. Ce dernier extrait présente une forme de discours «ces mots » requalifiée en contenu par la métaphore « rouge et noir » : le contenu n'est autre que les inférences que nous pouvons en tirer.

(6) Nous le savons tous d'expérience : quiconque communique à un public encore mal informé une nouvelle importante devient lui-même quelqu'un d'important. L'extinction du biotope terrestre, le grand hiver nucléaire, la collision de l'astéroïde qui dépeuplera la planète, l'autodestruction de l'existence humaine, la bombe climatique à retardement, le point de nonretour : ces mots rouge et noir se dégustent comme des sucres d'orge, d'autant mieux qu'ils aident, comme disait Gracq en 1940, à « triompher de l'angoissant par l'inouï ». Le vocabulaire cambré de l'ultime et de l'extrême a un pouvoir de dilatation jouxtant l'ivresse (DEBRAY : 45)

\section{En guise de conclusion}

Dans ce travail, volontairement limité, nous sommes arrivées à des conclusions qui, nous l'espérons, permettent de caractériser et donc de catégoriser par des oppositions objectives deux genres textuels.

- Dans le genre écrit scientifique, le scripteur réalise sa visée objectivante selon une homotopie de type centrifuge. Il rend accessible les cadres de l'entendement théorique au moyen de stratégies discursives particulières. Dans cette dynamique, le Topique en position saillante est généralement placé en titre, pour être ensuite projeté, expansé vers des espaces explicatifs ou descriptifs enchainés par des successions de reprises à l'identiques.

- Dans le genre essai polémique, l'auteur conceptualise, déforme ou manipule des données factuelles et inférentielles en les orientant vers un point d'attention créatif ou Focus selon une homotopie de type centripète.

Ce travail n'a pas la prétention d'avoir épuisé son objet et il faudrait l'examiner à la mesure des travaux récents sur le lexique des écrits scientifique ${ }^{7}$ menés par l'équipe de Grenoble et aussi par une lecture plus attentive des théories complémentaires relatives au topique et au focus.

\section{BIBLIOGRAPHIE}

ADAM Jean-Michel (2010), La notion de typologie de textes en didactique du français. Une notion « dépassée »?, Recherches $\mathrm{n}^{\circ} 42$, p. 11-23.

ACHARD-BAYle Guy (2008), Les Réalités Conceptuelles Identité et/en Fiction, Recherches textuelles $\mathrm{n}^{\circ} 8$, Metz, Université de Metz.

ACHARD-BAYLE Guy (2010), Du Cercle linguistique de Prague à une linguistique du texte 'à la française'. Plaidoyer pour une géopolitique des sciences, in : revue ALL, L'Analisi linguistica e litteraria, XVIII, Milan, Université Catholique, p. 431-436.

\footnotetext{
${ }^{7}$ En particulier dans le N ${ }^{\circ} 41$ de Lidil la proposition de Kjersti Fløttum et Eva Thue Vold «L'éthos auto-attribué d'auteurs-doctorants dans le discours scientifique » et celle de Agnès Tutin, «Dans cet article, nous souhaitons montrer que... Lexique verbal et positionnement de l'auteur dans les articles en sciences humaines» et dans le volume XII-2, décembre 2007, de la Revue Française de Linguistique Appliquée « Lexique et écrits scientifique » coordonnée par Agnès Tutin.
} 
En ligne : <http://www.educatt.it/libri/all/ALLPDF/201002BayleC.pdf>.

APOTHELOZ Denis (1995), Nominalisations, référents clandestins et anaphores atypiques, Travaux neuchâtelois de linguistique (TRANEL), no ${ }^{\circ} 23$, p. 143173.

BARTNING Inge (1996), Eléments pour une typologie des SN complexes en de en français, Langue française, ${ }^{\circ} 109$, p. 29-43.

COMBETTES Bernard (1977), Ordre des éléments de la phrase et linguistique du texte, Pratiques n ${ }^{\circ} 13$, p. 91-101.

COMBETTES Bernard (1988), Pour une grammaire textuelle: La progression thématique, De Boeck-Duculot $2^{\text {ème }}$ édition.

CONDAMINES Anne (2005), Anaphore nominale infidèle et hyperonymie : le rôle du genre textuel, Revue de Sémantique et Pragmatique, n ${ }^{\circ}$ 18, p. 23-42.

GuILlOT Céline (2007), Entre anaphore et deixis : l'anaphore démonstrative à fonction résomptive, in: D. TROTTER (éd.), Actes du XXIVe Congrès international de linguistique et de philologie romanes, Aberystwyth, (1-6 août) 2004, vol. 3, p. 307-315.

KARA Mohamed, WIEDERSPIEL Brigitte (2010), Choix lexicaux dans les reprises anaphoriques conceptuelles, in : Directions actuelles en linguistique du texte : Le Texte: modèles, méthodes, perspectives, Cluj-Napoca, Casa Cartii de Stiinta, p. 200-212.

KARA Mohamed, WIEDERSPIEL Brigitte (2011), Anaphore Résomptive Conceptuelle et mémoire discursive: entre identité et altérité, CENEL, Itinéraires LTC (Littérature, Textes, Cultures, p. 85-99.

KLEIBER Georges, Pour une explication du paradoxe de la reprise immédiate, Langue française, $\mathrm{n}^{\circ} 73,1986$, p. 54-79.

LE PESANT Denis (2002), La détermination dans les anaphores fidèles et infidèles, Langages, $\mathrm{n}^{\circ} 145$, p. 39-59.

PetitjeAn André (1989), Les typologies textuelles, Pratiques n62, p. 86-125.

PAVEAU Marie-Anne (2006), Les prédiscours. Sens, mémoire, cognition, Paris, Presses de la Sorbonne nouvelle.

SCHNEDECKER Catherine (2006). Anaphores prédicatives démonstratives: de la cohésion syntagmatique à la cohérence textuelle, CORELA - Organisation des textes et cohérence des discours. Publié en ligne le 27 octobre 2006 sous le lien : $\quad$ http://corela.edel.univ-poitiers.fr/index.php/index.php?id=1437. (Consulté le 3/09/2011).

TUTIN Agnès (2007), Traitement sémantique par analyse distributionnelle des noms transdisciplinaires des écrits scientifiques, TALN, Toulouse, 5-8 juin 2007.

VENIARD Marie (à paraître), Anaphores lexicales en contexte d'hétérogénéité énonciative et effets pragmatiques associés, Actes du colloque Ci-Dit IV: Discours rapporté, citation et pratiques sémiotiques. Nice 200.

WLODARCZYK André (2003), Sur les homotopies du topique et du focus, Ordre et distinction dans la langue et le discours, Paris, Champion, p. 513-526.

\section{Corpus :}

DEBRAY Régis, Du bon usage des catastrophes, Paris, Gallimard, 2011. 
Scientext : équipes de recherche : le LIDILEM (Université Stendhal, Grenoble III), le LiCorN (Université de Bretagne Sud), le LLS (de l'Université de Chambéry). Librement interrogeable sur : http://scientext.msh-alpes.fr. 